\title{
Recent Results On light Pseudoscalar Mesons
}

\section{Paolo Gauzzi*}

Università La Sapienza di Roma e INFN Sezione di Roma

E-mail: paolo.gauzzieromal.infn.it

The light pseudoscalar mesons are a powerful tool to investigate the Effective Field Theories that describe the strong interactions at low energies where the perturbative QCD is not applicable.

Recent results on $\pi^{0}$ and $\eta$ mesons are reviewed. The $\eta \rightarrow 3 \pi$ decay and the extraction of fundamental parameters like the masses of the light quarks is described. The measuremens of the Transition Form Factors in $\gamma \gamma$ processes and in Dalitz decays are compared with some theoretical predictions. Finally the search for Dark Photons in decays involving $\pi^{0}$ and $\eta$ is discussed.

The 26th International Nuclear Physics Conference

11-16 September, 2016

Adelaide, Australia

${ }^{*}$ Speaker. 


\section{Introduction}

In the Standard Model (SM) the strong interactions are described by Quantum Chromodynamics (QCD). At low energies the perturbative treatment of QCD cannot be applied, then effective field theories are needed to describe the strong interactions. The most successful of these theories is the Chiral Perturbation Theory (ChPT), in which the spontaneous breaking of the chiral symmetry $\left(\mathrm{SU}(3)_{L} \times \mathrm{SU}(3)_{R}\right)$ of the QCD Lagrangian gives rise to an octect of Nambu-Goldostone bosons, the pions, the kaons and the $\eta$ meson. Extensions of ChPT have been developed to include also the $\eta^{\prime}(958)$ meson. Then the study of these pseudoscalar mesons offers a unique opportunity to test the symmetries and the symmetry breaking mechanisms in QCD. Large samples of light pseudoscalar mesons are available from different sources: experiments at $e^{+} e^{-}$colliders, KLOE/KLOE-2 at the Frascati $\phi$-factory DA $\Phi N E$, BESIII at the Beijing collider BEPC-II, and the Novosibirsk experiments SND and CMD-3 at VEPP2000; fixed target experiments as WASA@COSY in Jülich; and also photoproduction experiments like CLAS at the Jefferson Lab and A2 at the Mainz microtron MAMI. In this review some recent results on processes involving $\eta$ and $\pi$ mesons are reported. In Sect. 2 the $\eta \rightarrow 3 \pi$ decay and the related information on the masses of the light quarks are shown; in Sect.3 some results on transition form factors both in the space-like and time-like region of momentum transfer are reported; Sect. 4 is dedicated to the searches of dark photons in processes with $\eta$ and $\pi^{0}$ mesons.

\section{2. $\eta \rightarrow 3 \pi$ and the light quark masses}

The $\eta \rightarrow 3 \pi$ decay is a strong process, isospin violating, since the electromagnetic contribution is strongly suppressed according to the Sutherland theorem[1]. This decay proceeds through a Lagrangian term proportional to the $d-u$ quark mass difference.

$$
\mathscr{L}=-\frac{1}{2}\left(m_{u}-m_{d}\right)(\bar{u} u-\bar{d} d)
$$

The decay amplitude is proportional to $Q^{-2}$, where the quadratic quark mass ratio is defined as:

$$
Q^{2}=\frac{m_{s}^{2}-\hat{m}^{2}}{m_{d}^{2}-m_{u}^{2}}
$$

with $\hat{m}=\frac{1}{2}\left(m_{u}+m_{d}\right) . Q=24.3$ can be obtained from a combination of pion and kaon masses, according to the Dashen theorem[2]. With this value, the decay width can be calculated at different orders of ChPT, $\Gamma_{L O}\left(\eta \rightarrow \pi^{+} \pi^{-} \pi^{0}\right)=66 \mathrm{eV}, \Gamma_{N L O}\left(\eta \rightarrow \pi^{+} \pi^{-} \pi^{0}\right)=160-210 \mathrm{eV}$, and $\Gamma_{N N L O}(\eta \rightarrow$ $\left.\pi^{+} \pi^{-} \pi^{0}\right)=230-270 \mathrm{eV}$, approaching very slowly to the experimental value $\Gamma\left(\eta \rightarrow \pi^{+} \pi^{-} \pi^{0}\right)=$ $(300 \pm 12) \mathrm{eV}[3]$. It is also known that the $\pi \pi$ final state interaction plays an important role.

The measurement of the Dalitz plot of $\eta \rightarrow \pi^{+} \pi^{-} \pi^{0}$ can provide stringent constraints on the light quark masses. The Dalitz plot distribution is expressed in terms of the normalized variables:

$$
X=\sqrt{3} \frac{T_{+}-T_{-}}{Q_{\eta}} \quad ; \quad Y=3 \frac{T_{0}}{Q_{\eta}}
$$

where the $T_{i}$ are the kinetic energies of the pions and $Q_{\eta}=m_{\eta}-2 m_{\pi_{ \pm}}-m_{\pi_{0}}$. The squared amplitude is usually parametrized as a Taylor expansion around the center:

$$
|A(X, Y)|^{2}=N\left(1+a Y+b Y^{2}+c X+d X^{2}+e X Y+f Y^{3}+g X^{2} Y+\ldots\right)
$$


Then the parameters are extracted from a fit of the Dalitz plot distribution to eq.(2.4). The Dalitz plot has been recently measured by KLOE with the decay $e^{+} e^{-} \rightarrow \phi \rightarrow \eta \gamma$ with $\eta \rightarrow \pi^{+} \pi^{-} \pi^{0}$, on a sample of $1.6 \mathrm{fb}^{-1}$ of data, corresponding to $4.7 \times 10^{6}$ events [4], improving the previous measurement based on a smaller data sample[5]. In fig.1 the Dalitz plot distribution is shown. The
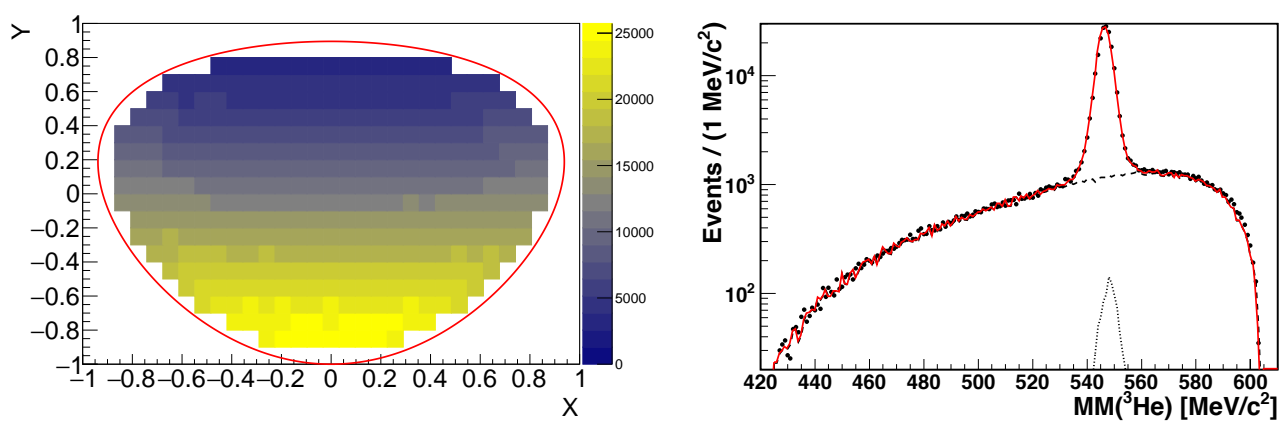

Figure 1: Left: KLOE Dalitz plot of $\eta \rightarrow \pi^{+} \pi^{-} \pi^{0}$ [4]; right: WASA, $p d \rightarrow^{3} H e \eta$, missing mass with respect to ${ }^{3} \mathrm{He}[7]$.

BESIII Collaboration exploited another radiative decay, $e^{+} e^{-} \rightarrow J / \psi \rightarrow \eta \gamma$ with $\eta \rightarrow \pi^{+} \pi^{-} \pi^{0}$, selecting about 80000 events from a sample of $1.31 \times 10^{9} \mathrm{~J} / \psi$ produced[6]. The Dalitz plot projections are shown in fig.2. The WASA measurement has been performed by using $1.74 \times 10^{5} \eta$ decays
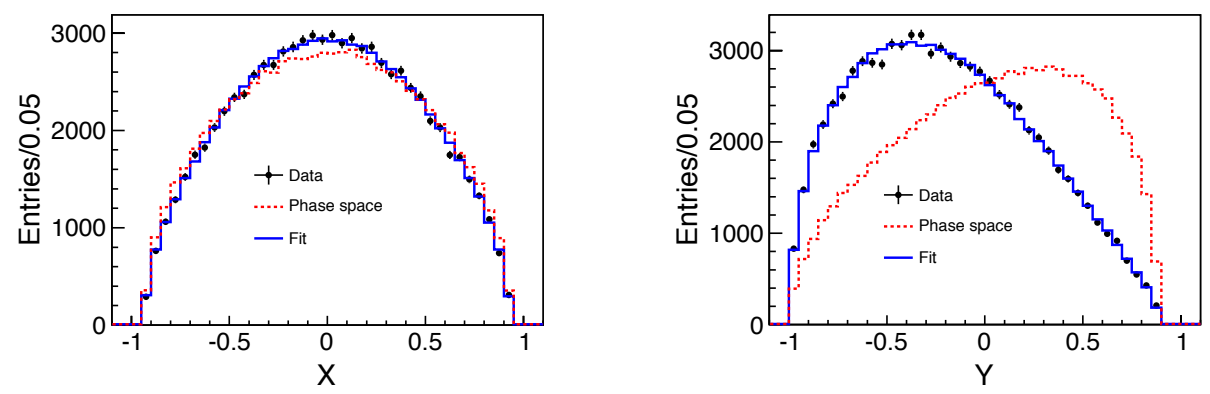

Figure 2: BESIII: Dalitz plot projections for $\eta \rightarrow \pi^{+} \pi^{-} \pi^{0}[6]$.

from the reaction $p d \rightarrow{ }^{3} \mathrm{He \eta}$, and the distribution of the missing mass with respect to the ${ }^{3} \mathrm{He}$ is reported in fig.1[7]. In tab.1 the Dalitz plot parameters are listed and compared to some theoretical predictions. The $c$ and $e$ parameters are $\mathrm{C}$-violating and are compatible with zero for all the experiments. Comparing the results the following conclusions can be drawn: (i) the experimental values of the parameters agree within the uncertainties; (ii) last KLOE measurement is the most precise at present and it is the only one sensitive to the $g$ parameter; (iii) naive ChPT is not able to reproduce all the parameters; (iv) better agreement is found with models which combine ChPT with a dispersive treatment of the $\pi \pi$ final state interaction (last two lines of tab.1).

The amplitude of the neutral decay $\eta \rightarrow 3 \pi^{0}$ is symmetric for the exchange of the pions, and is 


\begin{tabular}{|c|ccccc|}
\hline & $a$ & $b$ & $d$ & $f$ & $g$ \\
\hline KLOE ('08)[5] & $-1.090 \pm 0.020$ & $0.124 \pm 0.012$ & $0.057 \pm 0.017$ & $0.14 \pm 0.02$ & \\
WASA[7] & $-1.144 \pm 0.018$ & $0.219 \pm 0.051$ & $0.086 \pm 0.023$ & $0.115 \pm 0.037$ & \\
BESIII[6] & $-0.128 \pm 0.017$ & $0.153 \pm 0.017$ & $0.085 \pm 0.018$ & $0.173 \pm 0.035$ & \\
KLOE ('16)[4] & $-1.095 \pm 0.004$ & $0.145 \pm 0.006$ & $0.081 \pm 0.007$ & $0.141 \pm 0.011$ & $-0.044 \pm 0.016$ \\
\hline NNLO ChPT[8] & $-0.121 \pm 0.075$ & $0.394 \pm 0.102$ & $0.055 \pm 0.057$ & $0.025 \pm 0.160$ & \\
NREFT[9] & $-1.213 \pm 0.014$ & $0.308 \pm 0.023$ & $0.050 \pm 0.003$ & $0.083 \pm 0.019$ & $-0.039 \pm 0.002$ \\
JPAC[10] & $-1.117 \pm 0.035$ & $0.188 \pm 0.014$ & $0.079 \pm 0.003$ & $0.090 \pm 0.003$ & $-0.63 \pm 0.012$ \\
ChPT + KT eq.[11] & $-1.154 ;-1.142$ & $0.171 ; 0.202$ & $0.094 ; 0.097$ & $0.108 ; 0.123$ & $-088 ;-087$ \\
\hline
\end{tabular}

Table 1: $\eta \rightarrow \pi^{+} \pi^{-} \pi^{0}$ Dalitz plot parameters from experiments compared to some theoretical models.

generaly written in terms of the distance from the Dalitz plot center:

$$
Z=X^{2}+Y^{2}=\frac{2}{3} \sum_{i=1}^{3}\left(\frac{3 T_{i}}{Q_{\eta}}-1\right)^{2}
$$

where $Q_{\eta}=m_{\eta}-3 m_{\pi^{0}}$. The amplitude parametrization is:

$$
|A(Z)|^{2}=N(1+2 \alpha Z+\ldots)
$$

The slope parameter $\alpha$ is extracted from a fit to the $Z$ distribution, as shown in fig. 3 for the two most recent measurements. The left plot refers to KLOE, $e^{+} e^{-} \rightarrow \phi \rightarrow \eta \gamma$ with $\eta \rightarrow 3 \pi^{0}$ from a sample
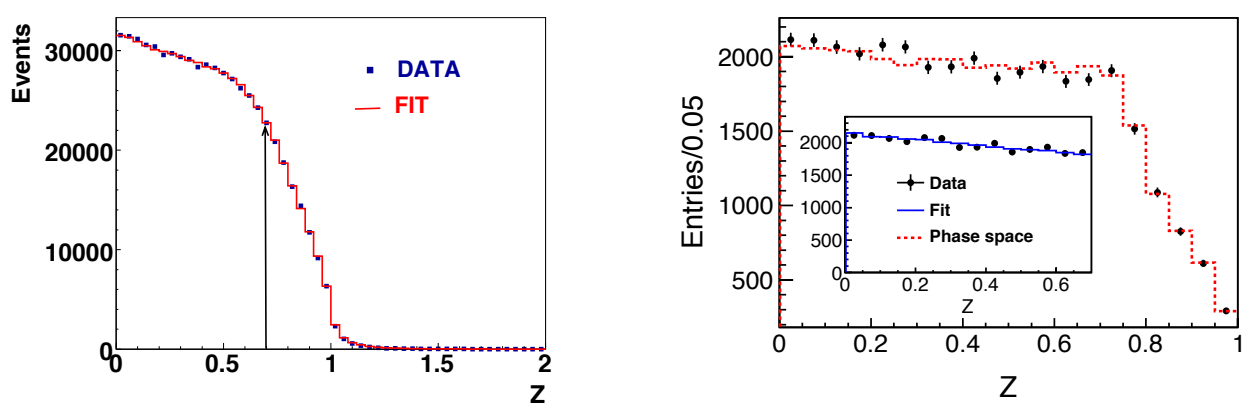

Figure 3: $Z$ distribution for $\eta \rightarrow 3 \pi^{0}$ : left: KLOE[12], right: BESIII[6].

of $420 \mathrm{pb}^{-1}$, corresponding to $5 \times 10^{5}$ events: from the fit the slope $\alpha=-0.0301 \pm 0.0035_{+0.0035}^{+0.0022}$ is extracted[12]. The right plot is from BESIII, from about 34000 events $e^{+} e^{-} \rightarrow \phi \rightarrow J / \psi \rightarrow \eta \gamma$ with $\eta \rightarrow 3 \pi^{0}$, resulting in $\alpha=-0.055 \pm 0.014 \pm 0.004[6]$. A summary plot of the slope measurements compared to some theoretical calculations is shown in fig.4. Naive ChPT predicts only positive $\alpha$ values, while dispersive approaches agree with the experimental data.

Dispersive methods make use of fits to the experimental Dalitz plots to derive some subtraction costants and to obtain the $Q$ ratio. In the most recent paper on this subject Colangelo and collaborators[13] fit the KLOE Dalitz plot of $\eta \rightarrow \pi^{+} \pi^{-} \pi^{0}$ to obtain $Q=22.0 \pm 0.7$, far from the Dashen value 24.3, and using the ratio $\frac{m_{s}}{\hat{m}}=27.30 \pm 0.34$ from Lattice QCD the authors were able to determine the $u / d$ quark mass ratio $\frac{m_{u}}{m_{d}}=0.44 \pm 0.03$. 


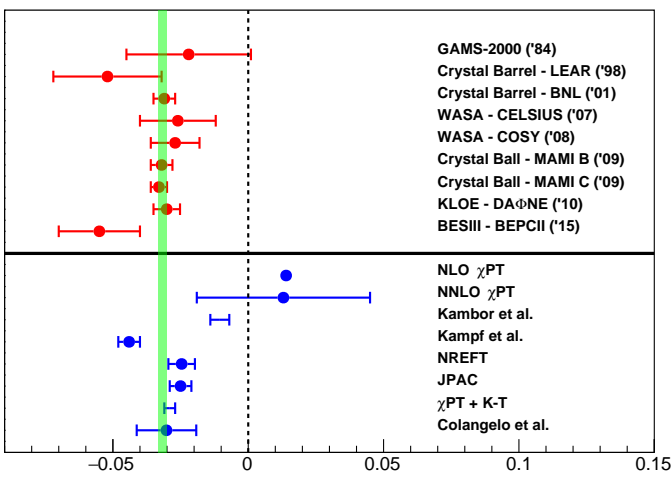

Figure 4: Slope of the $\eta \rightarrow 3 \pi^{0}$ Dalitz plot: measurement compared to some theoretical determinations.

\section{Transition Form Factors}

The Transition Form Factors (TFFs) describe the coupling of mesons to photons and provide information about the nature of the mesons and their structure. Recently the interest in the TFFs has been renewed due to the discrepancy of more than three standard deviation between the theoretical and the experimental value of the anomalous magnetic moment of the muon. The main contribution to the hadronic part of the muon anomaly is the vacuum polarization, but the second one in order of relevance comes from the hadronic Light-by-Light (HLbL) scattering diagram (fig.5). The leading contribution to the HLbL is the single pseudoscalar exchange (fig.5), in which the TFFs appear at the two vertices of the meson propagator. The calculation of this contribution is model dependent
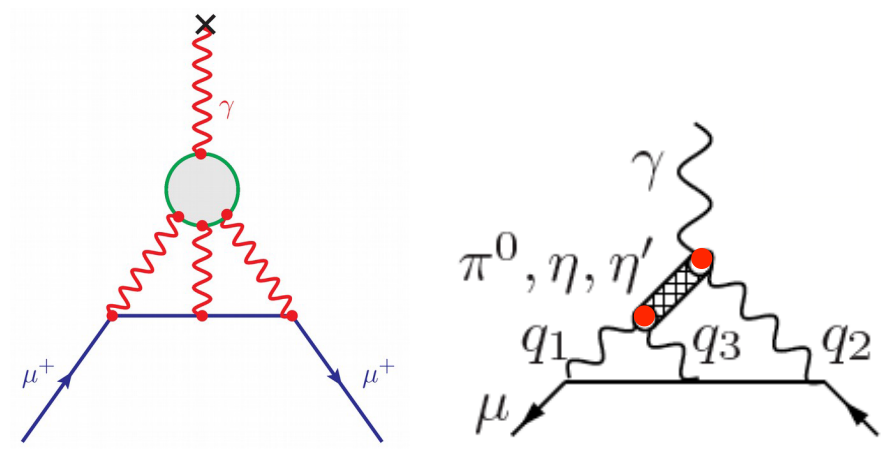

Figure 5: Left: hadronic Light-by-Light scattering; right: single pseudoscalar exchange in HLbL.

since the exchanged meson is off-shell, and the TFFs for off-shell meson are not measurable quantities. Nevertheless any experimental information the TFFs, both for space-like and time-like $q^{2}$, can help in constraining the models used in the calculations[14].

The TFFs for space-like $q^{2}$ can be measured by means of the $\gamma \gamma$ processes like $e^{+} e^{-} \rightarrow e^{+} e^{-} \gamma^{\star} \gamma^{\star} \rightarrow$ $e^{+} e^{-} P$, where $P$ is a pseudoscalar meson. Different kind of measurements are possible: (i) No Tag, when the scattered $e^{+}$and $e^{-}$escape the detector, the virtual photon are quasi-real $\left(q_{i}^{2} \simeq 0\right)$, the 
radiative width of the meson $\Gamma(P \rightarrow \gamma \gamma)$ is accessible in this case; (ii) Single Tag, when one of the scattered leptons is detected, so that the TFF as a function of the corresponding $Q^{2}=-q^{2}$ $\left(F\left(Q^{2}, 0\right)\right)$ can be measured; and (iii) Double Tag, when both the $e^{+}$and the $e^{-}$are detected, the cross-sections are very small, unless some specific devices a low polar angle, the $\gamma \gamma$ taggers, are used. As an example of No Tag, KLOE studied the process $e^{+} e^{-} \rightarrow e^{+} e^{-} \gamma^{\star} \gamma^{\star} \rightarrow e^{+} e^{-} \eta$, with $\eta \rightarrow \pi^{+} \pi^{-} \pi^{0}$ and $\eta \rightarrow 3 \pi^{0}$, by using $240 \mathrm{pb}^{-1}$ of data collected at $\sqrt{s}=1 \mathrm{GeV}$, off the $\phi$ peak to avoid the background from $\phi$ decays. The cross-section $\sigma\left(e^{+} e^{-} \rightarrow e^{+} e^{-} \eta\right)=(32.7 \pm 1.3 \pm 0.7)$ $\mathrm{pb}$ has been measured and the decay width $\Gamma(\eta \rightarrow \gamma \gamma)=(520 \pm 20 \pm 13) \mathrm{eV}$ has been derived[15]. The most recent Single Tag measurements have been performed at the B-factories PEP-II and KEKB, by the BaBar and Belle. The $\pi^{0}$ TFF (fig.6) shows a discrepancy between BaBar and Belle data, while the latter are compatible with the asymptotic QCD expected behaviour, the former clearly deviate from that behaviour. Moreover the low energy region $Q^{2} \lesssim 1 \mathrm{GeV}^{2}$, which is relevant to constrain the TFF parametrizations for the HLbL calculation, has not been yet explored. BESIII is analyzing the data collected at the $\psi(3770)$ peak to cover the region $0.3<Q^{2}<3 \mathrm{GeV}^{2}$. Before the start of the KLOE-2 run, the KLOE Collaboration installed two tagging devices: the Low Energy Tagger (LET)[16], consisting of two crystal calorimeters placed at about $1 \mathrm{~m}$ from the DAФNE interaction (IP) point to detect scattered $e^{ \pm}$of $E \simeq 150-350 \mathrm{MeV}$, and the High Energy Tagger, two scintillator hodoscopes placed at about $11 \mathrm{~m}$ from the IP to detect the $e^{ \pm}$in the range $E \simeq 420-495 \mathrm{MeV}$ [17]. If both $e^{+}$and $e^{-}$are detected in the two HET stations $Q_{1,2}^{2} \simeq 0$ and the $\Gamma\left(\pi^{0} \rightarrow \gamma \gamma\right)$ will be obtained. If on the other hand one lepton is detected in the HET and the other one in the KLOE main detector, $F\left(Q^{2}, 0\right)$ for $Q^{2}<0.1 \mathrm{GeV}^{2}$ will be measured (fig.6). Also BESIII
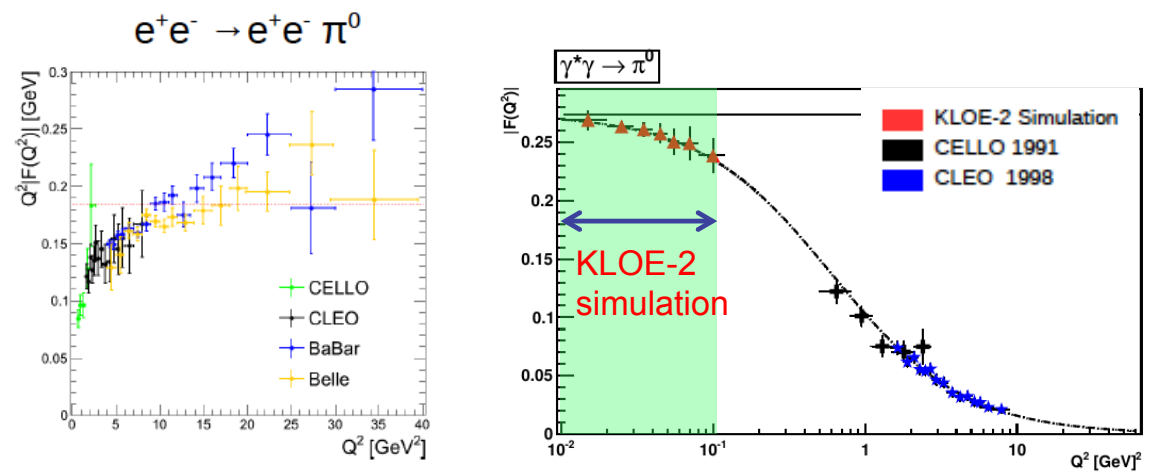

Figure 6: $\pi^{0}$ space-like TFF; left: high $Q^{2}$ behaviour; right: expectation at low $Q^{2}$ from KLOE-2.

will install two $\mathrm{PbWO}_{4}$ calorimeters at very small angles to be used as taggers in a next run. The TFFs for time-like $q^{2}$ can be studied by means of the Dalitz decays $P \rightarrow V \ell^{+} \ell^{-}$or $V \rightarrow P \ell^{+} \ell^{-}$, as functions of the invariant mass $q^{2}=m^{2}\left(\ell^{+} \ell^{-}\right)$. According to Vector Meson Dominance (VMD) are usually parametrized as $F\left(q^{2}\right)=1 /\left(1-\frac{q^{2}}{\Lambda^{2}}\right)$, where $\Lambda$ is a characteristic mass parameter. In tab.2 the recent results on the TFF measurements in Dalitz decay are listed. The measured slopes, $\Lambda^{-2}$, are in agreement within the errors with VMD predictions apart from the process $\omega \rightarrow \pi^{0} \mu^{+} \mu^{-}$. KLOE studied the process $\phi \rightarrow \eta e^{+} e^{-}$with $\eta \rightarrow 3 \pi^{0}$, by selecting events with 2 charged tracks and 6 prompt photons, and obtained the $\operatorname{Br}\left(\phi \rightarrow \eta e^{+} e^{-}\right)=(1.075 \pm 0.007 \pm 0.038) \times 10^{-4}[23]$. From the fit to the $e^{+} e^{-}$invariant mass distribution obtained the TFF shown in fig.7. The slope is 


\begin{tabular}{|c|c|c|}
\hline & $\Lambda^{-2}\left[\mathrm{GeV}^{-2}\right]-$ expt. & $\Lambda^{-2}\left[\mathrm{GeV}^{-2}\right]-$ VMD \\
\hline$\eta \rightarrow e^{+} e^{-} \gamma / \mu^{+} \mu^{-} \gamma$ & & \\
\hline $\mathrm{A} 2[18]$ & $1.95 \pm 0.15 \pm 0.10$ & 1.83 \\
NA60 (In-In)[19] & $1.95 \pm 0.17 \pm 0.04$ & \\
NA60 (p-A)[20] & $1.934 \pm 0.067 \pm 0.050$ & \\
\hline$\pi^{0} \rightarrow e^{+} e^{-} \gamma$ & & 1.7 \\
\hline NA62[21] & $2.02 \pm 0.31$ & \\
A2[22] & $1.65 \pm 0.55$ & 1.68 \\
\hline$\omega \rightarrow \pi^{0} \mu^{+} \mu^{-}$ & & \\
\hline NA60 (In-In)[19] & $2.24 \pm 0.06 \pm 0.02$ & \\
NA60 (p-A)[20] & $2.223 \pm 0.026 \pm 0.037$ & \\
\hline
\end{tabular}

Table 2: Slopes of TFFs from Dalitz decays.

$\Lambda^{-2}=(1.28 \pm 0.10 \pm 0.09) \mathrm{GeV}^{-2}$ compatible with VMD prediction $\Lambda_{V M D}^{-2} \simeq 1 \mathrm{GeV}^{-2}$. KLOE also measured $\phi \rightarrow \pi^{0} e^{+} e^{-}$, by selecting events with two tracks and two prompt photons, and obtained $\operatorname{Br}\left(\phi \rightarrow \pi^{0} e^{+} e^{-}\right)=\left(1.35 \pm 0.05_{-0.10}^{+0.05}\right) \times 10^{-5}$. The slope of the TFF turns out to be $\Lambda^{-2}=(2.02 \pm 0.11) \mathrm{GeV}^{-2}$ [24], which does not agree with VMD, as shown in fig.7.
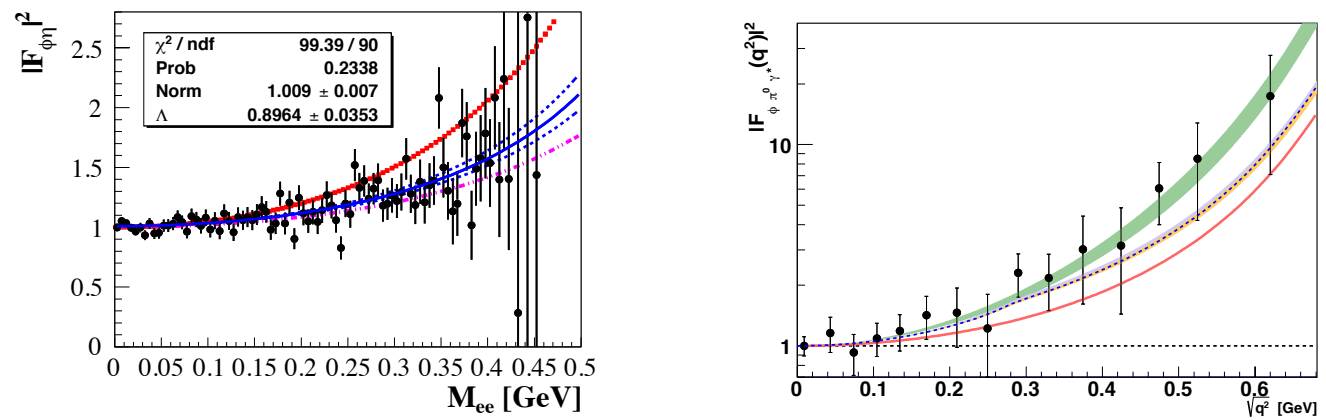

Figure 7: TFFs: left $-\phi \rightarrow \eta e^{+} e^{-}$, the blue line is the VMD prediction[23]; right $-\phi \rightarrow \pi^{0} e^{+} e^{-}$, the red line is the VMD prediction[24].

\section{Dark Photon searches}

Dark Matter (DM) existence is widely accepted, it should account for about $25 \%$ of the total energy density of the Universe. Several models have been proposed in which DM consists of new particles belonging to a secluded gauge sector under which the Standard Model particles are uncharged. In some of these models the new interaction is mediated by a massive gauge vector boson, the $U$ boson (often called also $\mathrm{A}^{\prime}$ or $\gamma^{\prime}$ ) which can be kinematically mixed with the ordinary photon (fig.8). The existence of a $U$ boson of mass of $O(1 \mathrm{GeV})$ and mixing parameter $\varepsilon$ in the range $10^{-2} \div 10^{-7}$ has been proposed to explain the astrophysical anomalies observed by several experimental Collaborations (like AMS02, PAMELA, INTEGRAL, FERMI, DAMA)[25].

KLOE used the same decay $\phi \rightarrow \eta e^{+} e^{-}$already exploited for the TFFs to look for a $U$ boson signal as a spike in the dilepton invariant mass distribution $\left(U \rightarrow e^{+} e^{-}\right)$[26]. The decay $\pi^{0} \rightarrow e^{+} e^{-} \gamma$ has 
been studied by WASA[27], HADES[28], and more recently by NA48/2[29]. No peaks have been found in the dilepton invariant mass, and limits on the mixing parameters have been obtained. A summary plot showing the exclusion region as a function of the $U$ boson mass is shown in fig.8.
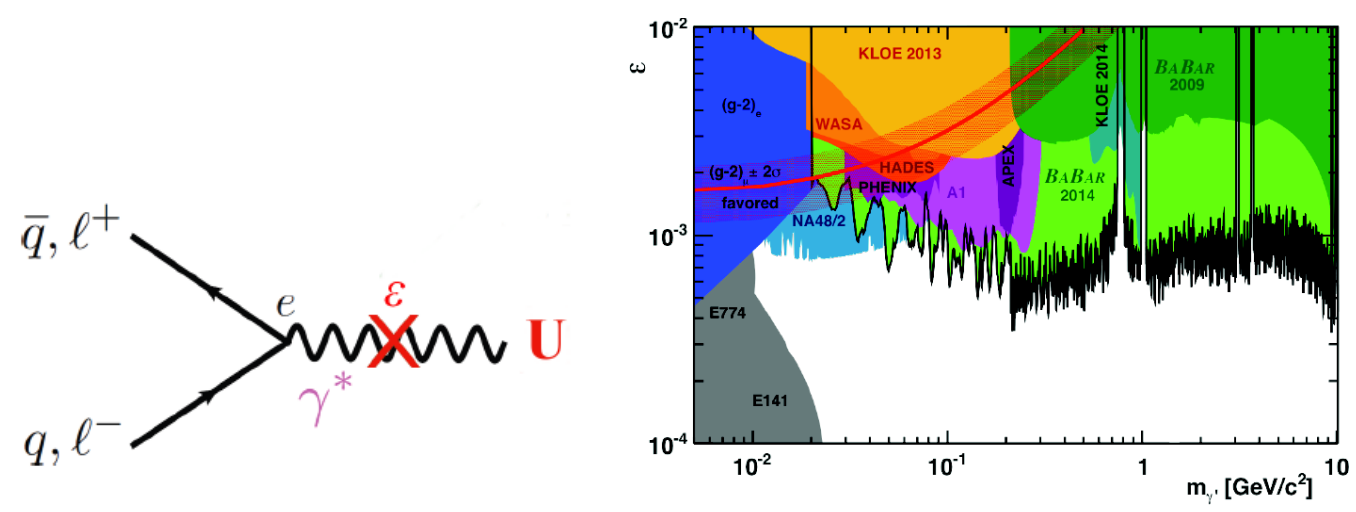

Figure 8: Left: mixing mechanism of the $U$ boson with the ordinary photon; right: exclusion plot of the mixing parameter as a function of the $U$ boson mass.

\section{Conclusions}

The study of the light pseudoscalar mesons is a still alive research field. It allows precision tests of Effective Field Theories, like the Chiral Perturbation Theory. These precision measurements at low energy are also a powerful tool to investigate the presence of dark matter particles. Large samples of pseudoscalar mesons are available, being produced at $e^{+} e^{-}$colliders, in fixed target experiments, and in photoproduction experiments. More data will be available in the next future, as the KLOE-2 Collaboration is taking data at DA $\Phi N E$ with the goal to collect $5 \mathrm{fb}^{-1}$ by mid of 2018, BESIII is also in data-taking phase, and there are other experiments planned at JLAB.

\section{References}

[1] D.G.Sutherland, Current algebra and the decay $\eta \rightarrow 3 \pi$, Phys.Lett. 23384 (1966).

[2] R.F.Dashen, Chiral $S U(3) \times S U(3)$ as a symmetry of the strong interactions, Phys.Rev. 1831245 (1969).

[3] C.Patrignani et al., The Review of Particle Physics, Chin. Phys. C40 100001 (2016).

[4] A.Anastasi et al., Precision measurement of the $\eta \rightarrow \pi^{+} \pi^{-} \pi^{0}$ Dalitz plot distribution with the KLOE detector, JHEP 1605019 (2016) [arXiv:1601.06985].

[5] F.Ambrosino et al., Determination of $\eta \rightarrow \pi^{+} \pi^{-} \pi^{0}$ Dalitz plot slopes and asymmetries with the KLOE detector, JHEP 0805006 (2008) [arXiv:0801.2642].

[6] M.Ablikim et al., Measurement of the matrix elements for the decays $\eta \rightarrow \pi^{+} \pi^{-} \pi^{0}$ and $\eta / \eta^{\prime} \rightarrow \pi^{0} \pi^{0} \pi^{0}$, Phys.Rev. D92 012014 (2015) [arXiv:1506.05360].

[7] P.Adlarson et al., Measurement of the $\eta \rightarrow \pi^{+} \pi^{-} \pi^{0}$ Dalitz plot distribution, Phys.Rev. $\mathbf{C 9 0} 045207$ (2014) [arXiv:1406.2505]. 
[8] J.Bijnens, K.Ghorbani, $\eta \rightarrow 3 \pi$ at Two Loops In Chiral Perturbation Theory, JHEP 0711030 (2007) [arXiv:0709.0230].

[9] S.P.Schneider et al., Rescattering effects in $\eta \rightarrow 3 \pi$ decays, JHEP 1102028 (2011) [arXiv:1010.3946].

[10] S.Guo et al., Three-body final state interaction in $\eta \rightarrow 3 \pi$ revisited, arXiv:1608.01447.

[11] B.Moussallam, M.Albaladejo, Role of the $a_{0}(980), f_{0}(980)$ resonances in $\eta \rightarrow 3 \pi$ from the Khuri-Treiman formalism, EPJ Web of Conferences 13003007 (2016).

[12] F.Ambrosino et al., Measurement of the $\eta \rightarrow 3 \pi^{0}$ slope parameter $\alpha$ with the KLOE detector, Phys.Lett. B649 16 (2011) [arXiv:1004.1319].

[13] G.Colangelo et al., $\eta \rightarrow 3 \pi$ : Study of the Dalitz Plot and Extraction of the Quark Mass Ratio Q, Phys.Rev.Lett. 118022001 (2017) [arXiv:1610.03494].

[14] F.Jegerlehner, A.Nyffeler, The Muon g-2, Phys.Rept. 4471 (2009) [arXiv:0902.3360].

[15] D.Babusci et al., Measurement of $\eta$ meson production in $\gamma \gamma$ interactions and $\Gamma(\eta \rightarrow \gamma \gamma)$ with the KLOE detector, JHEP 1301119 (2013) [arXiv:1211.1845].

[16] D.Babusci et al., The Low Energy Tagger for the KLOE-2 experiment, Nucl.Instr.and Meth. A617 81 (2010) [arXiv:0906.0875].

[17] F.Archilli et al., $\gamma \gamma$ tagging system for KLOE2 experiment, Nucl.Instr.and Meth. $\mathbf{A 6 1 7} 266$ (2010).

[18] H.Berghauser et al., Determination of the $\eta$-transition form factor in the $\gamma p \rightarrow p \eta \rightarrow p \gamma e^{+} e^{-}$ reaction, Phys.Lett. B701 562 (2011).

[19] R.Arnaldi et al., Study of the electromagnetic transition form-factors in $\eta \rightarrow \mu^{+} \mu^{-} \gamma$ and $\omega \rightarrow \mu^{+} \mu^{-} \pi^{0}$ decays with NA60, Phys.Lett. B677 260 (2009) [arXiv:0902.2547].

[20] R.Arnaldi et al., Precision study of the $\eta \rightarrow \mu^{+} \mu^{-} \gamma$ and $\omega \rightarrow \mu^{+} \mu^{-} \pi^{0}$ electromagnetic transition form-factors and of the $\rho \rightarrow \mu^{+} \mu^{-}$line shape in NA60, Phys.Lett. B757 437 (2016) [arXiv:1608.07898].

[21] C.Lazzeroni et al., Measurement of the $\pi^{0}$ Electromagnetic Transition Form Factor Slope, arXiv:1612.08162.

[22] P.Adlarson et al., Measurement of the $\pi^{0} \rightarrow e^{+} e^{-} \gamma$ Dalitz decay at MAMI, arXiv:1611.04739.

[23] D.Babusci et al., Study of the Dalitz decay $\phi \rightarrow \eta e^{+} e^{-}$with the KLOE detector, Phys.Lett. B742 1 (2015) [arXiv:1409.4582].

[24] A.Anastasi et al., Measurement of the $\phi \rightarrow \pi^{0} e^{+} e^{-}$transition form factor with the KLOE detector, Phys.Lett. B757 362 (2016) [arXiv:1601.06565].

[25] N.Arkani-Hamed et al., A theory of dark matter, Phys.Rev. D79 015014 (2009) [arXiv:0810.0713]; M.Pospelov et al., Secluded WIMP Dark Matter, Phys.Lett. B662 53 (2008) [arXiv:0711.4866].

[26] D.Babusci et al., Limit on the production of a light vector gauge boson in $\phi$ meson decays with the KLOE detector, Phys.Lett. B720 111 (2013) [arXiv:1210.3927].

[27] P.Adlarson et al., Search for a dark photon in the $\pi^{0} \rightarrow e^{+} e^{-} \gamma$ decay, Phys.Lett. B726 187 (2013) [arXiv:1304.0671].

[28] G.Agakishiev et al., Searching a Dark Photon with HADES, Phys.Lett. B731 265 (2014) [arXiv:1311.0216].

[29] J.R.Batley et al., Search for the dark photon in $\pi^{0}$ decays, Phys.Lett. B746 178 (2015) [arXiv:1504.00607]. 